\title{
An Alternative Implementation of Indicator Kriging on Mineral Reserves 3D Modeling
}

\author{
Zhanglin $\mathrm{Li}^{1}$ and Ping Wang ${ }^{2 *}$ \\ ${ }^{1}$ Computer Faculty, China University of Geosciences, Wuhan Hubei, 430074, China; ${ }^{2}$ Computer Faculty, China Univer- \\ sity of Geosciences, Wuhan Hubei, 430074, China
}

\begin{abstract}
Operating complexity, especially in the determination of cut-off values and the associated spatial variation, has been a long time standing problem for indicator kriging (IK) mineral reserves estimation method. In order to solve this problem, this text proposes an alternative method to implement IK reserves estimation in computer software. In this solution, threshold values of IK are dynamically selected according to the participant samples. Additionally, it considers the sharp of corresponding indicator variograms can be represented by the median indicator variogram. Thus, this method can theoretically improve the operational efficiency while reducing complexity of operation comparing with the classical IK method. A typical application based on a real developing mine was carried out to examine the practical validation of the proposed method. As a result, the simplified solution is not only easier to be utilized in practice but also identical in the accuracy and precision of estimates comparing with the traditional method. Therefore, it is reasonable to conclude that the proposed method is valid in both simplifying the operation process and improving calculation efficiency of traditional IK mineral reserve estimation method.
\end{abstract}

Keywords: Geo-information, mineral reserve estimation, spatial interpolation.

\section{INTRODUCTION}

The accuracy and precision of mineral reserves estimates are crucial in the context of severe competition on natural resources nowadays. As two typical mineral reserve estimation method, inverse distance weighting (IDW) and ordinary kriging $(\mathrm{OK})$ are usually practicable and effective at present in most cases [1,2]. IDW method has the advantages of high computation speed and convenient operation, but the calculated result is likely to be quite different with the real situation in most instances especially when mineralization is discontinuous or the ore-body is strongly affected by the later tectonism. OK which is a more advanced method compared with on IDW has a significant advantage that both continuity and variety of characters of deposit under study can be properly considered in spatial scale by using variogram. Thus the later method has a unique predominance on evaluating the statistic distribution of ore grades within an ore-body space. However, OK cannot guarantee credibility of the ore grade estimates if the sample dataset takes on an abnormal distribution which is unfortunately a common property of geological data.

A variant of OK, indicator kriging (IK) [3-5], based on a non-parametric transformation on original samples, is suitable for the non-normal distribution situations. Traditional IK requires lots of manual interactions in the indicator spatial variation modeling and much CPU time in the interpolation process. Thus the practicability of IK is far less than
IDW and OK although the associated validity is theoretically approved especially in the condition of non-normal distribution of sample data.

In this context, this article will firstly discuss and analyze on the basic principles of mineral resource estimation method and IK technology. Secondly, an optimized solution to the traditional IK method is proposed and the associated flow chart of calculating ore reserves by using the novel method is designed and succeeds in the algorithm implement in a geoscience information system. Thirdly, a case study based on a real mine dataset was carried out and illustrated, from which it can be concluded that the proposed method is valid and practicable through comparison with true situations and estimates resulted from other typical interpolation methods.

\section{BACKGROUND THEORIES}

\subsection{Mineral Reserve Estimation}

Estimation process of common mineral resources or reserves can be expressed as follows:

$$
\begin{aligned}
& Q=V D \\
& P=Q C
\end{aligned}
$$

where $\mathrm{V}$ and $\mathrm{C}$ respectively denotes the volume and the average ore grade of an ore block; D stands for the average weight of ore in the ore block; P and Q denotes respectively ore tonnage and metal tonnage.

From expression (1) and (2), it is clear that the basic parameters in mineral reverses estimation are respectively the 
volume, density and ore grade of an ore block. Among all of these parameters, the most crucial one is the average ore grade, which can directly affected the precision of mineral reserves estimates. Thus, how to obtain a credible average ore-grade of an ore block is a key problem in mineral reserve estimation.

\subsection{Indicator Kriging}

Indicator kriging (IK) is a representative non-parametric estimation method in geo-statistics. Compared with ordinary kriging, IK does not rely on stationary assumption of space phenomenon and is not in need of obeying a certain distribution of the regionalized variable under study [6]. Thus, with regard to the IK mineral reserves estimation, it is unnecessary to get rid of outlier in the raw data. The main objective for IK is to assess accurately uncertainty of un-sampled points measured by a random function through nonparametric ways.

A typical process of IK interpolation can be summarized in the following steps [7]:

1). Determination of cut-off values, including its total number and specific values.

2). Indicator transformation of original sample data to obtain indicator samples;

3). Calculation of indicator spatial variations corresponding to the series of indicator samples;

4). Estimation of conditional cumulated distributing function(CCDF) for every estimated ore block by OK based on the indicator sample data and the associated spatial variation;

5). Interpolation of estimated ore block based on the CCDFs estimates. The complete mineral reserves estimation information can be calculated by the expression (1) and (2).

As a nature technology in theory, more detailed principle and operator processes about IK will not be further illustrated in this paper. Anyone who is interested in these can find more detail in references [8-10].

What's notable about the above process of traditional IK mineral reserves estimation method are as follows: (1) its operation is quite complex because the determination of threshold values is lack of strict theoretical reference; (2) computation and fitting process of indicator variograms associated with all of the cut-off values is troublesome and will consume a large quantity of CPU-wise. Thus overcoming this two shortages in the implementation process of IK mineral reserves estimation is crucial, which is also a focus of this paper and will be discussed blow.

\section{MAIN PRINCIPLES AND IMPLEMENTATION}

Based on the analysis and discuss on the fundamental theory of IK method, it is not difficult to conclude that the global threshold values employed in traditional solution may not be a perfect idea. And thus in the specific implementation process of IK mineral reserves estimation in this study, the cut-off values is dynamically selected and calculated by the following expression:

$$
i\left(x ; z_{k}\right)=\left\{\begin{array}{l}
1, z(x) \leq z_{k} \\
0, z(x)>z_{k}
\end{array}, k=1,2,3, \cdots, n\right.
$$

where $z_{k}$ is the $\mathrm{k}$-th grade value of a participant sample during IK interpolation process corresponding to the unknown position $(\mathrm{x}) ; i\left(x ; z_{k}\right)$ is the indicator values associated with the current cutoff $z k$ at unknown position (x); n represents the total number of the participant samples.

As shown in expression (3), the global indicator sample values can be simply calculated by the participant samples within the searching neighborhood. As such, in the following estimation process, the indicator variogram associated with each threshold values can be supposed to be similar with the median indicator variogram, which is also the basic idea of median indicator kriging (MIK). Therefore, the determination of cutoff values and the CPU time consumption in indicator variogram computation and theoretical fitting can be almost considered as out of question for the new method.

As such, a flow chart associated with the optimized IK mineral reserves estimation discussed above is designed and illustrated in Fig. (1), from which the determination of many cutoff values is eliminated compared with traditional IK mineral reserves estimation methods.

\section{CASE STUDY}

\subsection{Study Area}

The experiment dataset is collected from a real developing mine in southeast China. It is a typical minerogenic deposit and the boundary line between ore bodies and the surrounding rock is vague and ambiguous. Additionally, sample grades present a well continuity and thus it is adaptive to apply geo-statistical kriging in this study area.

\subsection{Experimental Method Design}

Combining with the process of geo-statistical reserves estimation and characteristics of IK algorithm, in the process of implement IK algorithm in the study area, a detailed flow chart is proposed in Fig. (2) to carry out this case study.

In order to objectively evaluate the overall performance of the proposed method, cross validation and comparing with other similar technologies, including IDW, OK and traditional IK, are respectively applied in this test.

For cross validation procedure, all of the sampled points will be estimated by an assigned method and thus it can result in both estimated and measured grade values associated with every point in the dataset, which are crucial to assess the accuracy or error for the interpolation method. Additionally, in order to quantitatively evaluate performance of the interpolation result corresponding to some certain power value, a measure named Root Mean Square Error (RMSE) resulting from cross validation is employed, which is calculated by:

$$
\mathrm{RMSE}=\sqrt{\frac{1}{\mathrm{~N}} \sum_{i=1}^{\mathrm{N}}\left(\frac{z\left(x_{i}\right)-z^{*}\left(x_{i}\right)}{z\left(x_{i}\right)}\right)^{2}}
$$




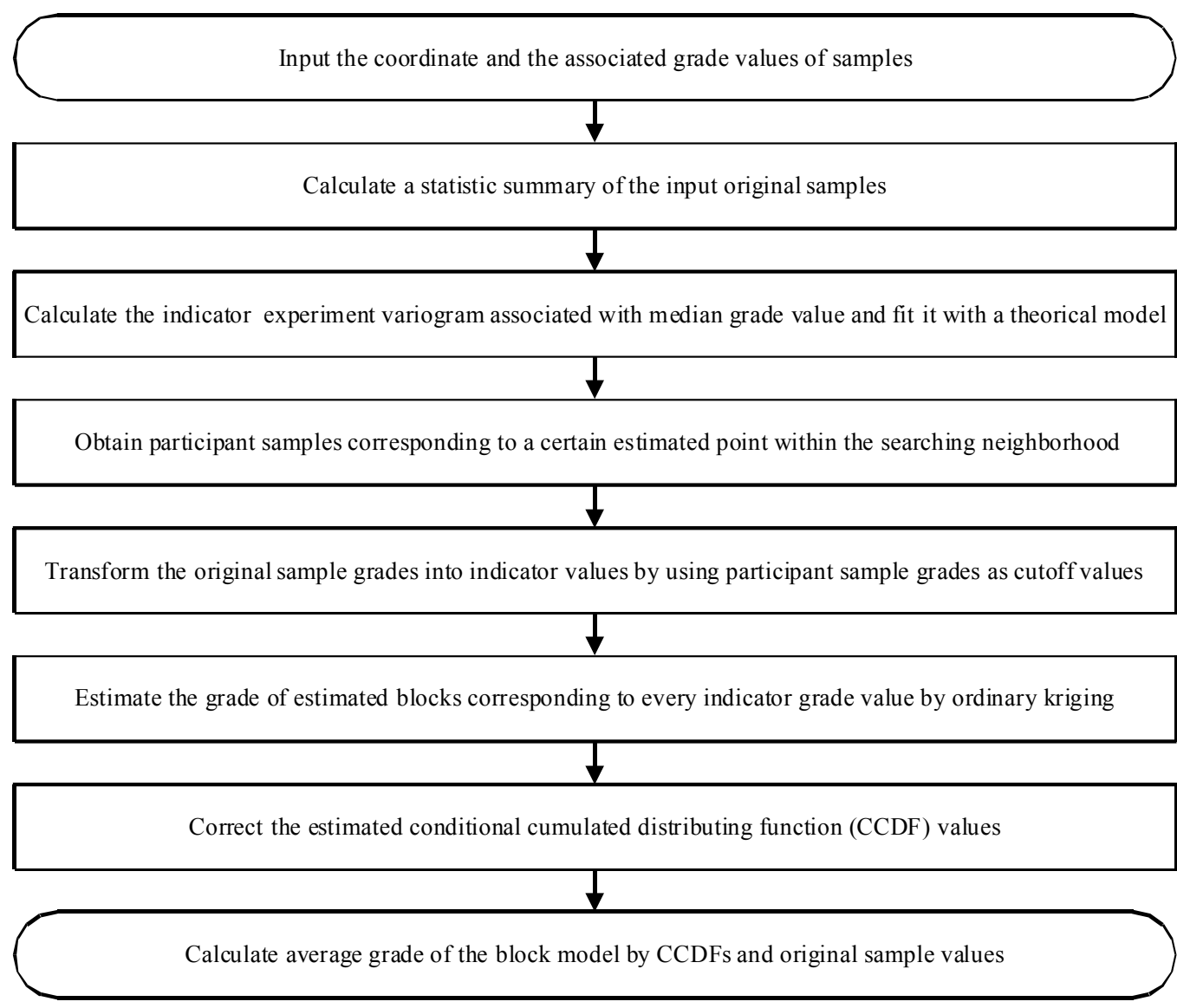

Fig. (1). A typical main flow chart of implementation on IK mineral reserves estimation method.

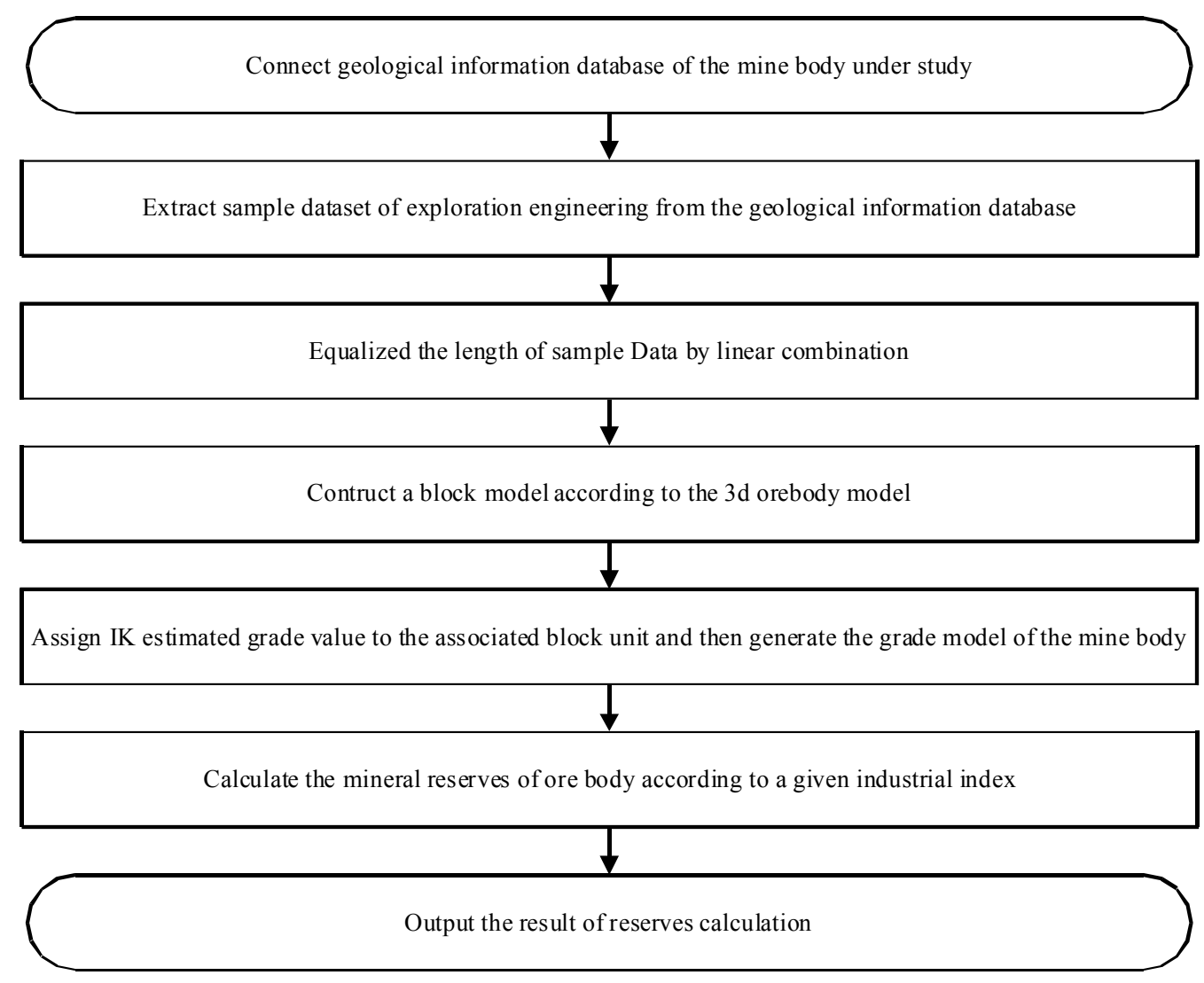

Fig. (2). A typical full procedure to perform IK mineral reserves estimation in the case study. 
where $\mathrm{N}$ represents the number of the points to be estimated, $z^{*}\left(x_{i}\right)$ and $z\left(x_{i}\right)$ represents the estimate and true attribute value of the interpolation point.

During the interpolation process in this test, radius of the searching neighborhood is limited within $150 \mathrm{~m}$ to guarantee that the quantity of participant samples is enough. All of the four kinds of interpolators (IDW, OK, IK and optimized IK) adopt the same searching radius to produce a relatively objective test result.

\subsection{Experimental Procedure}

(1) Data acquisition and preprocessing

In this test, sample data acquisition and preprocessing process can be generalized as the following three steps:

Firstly connect the constructed geological database and select a correct exploration area. According to the basic statistic characters expressed by Fig. (3), the sample data significantly deviates from normal distribution.

Secondly, the sample dataset including spatial positions and grades from exploration engineering, for instance drillings, are imported in this algorithm.

Thirdly, transform the sample data into an equalization length. In this test, this length is assigned as $3(\mathrm{~m})$.

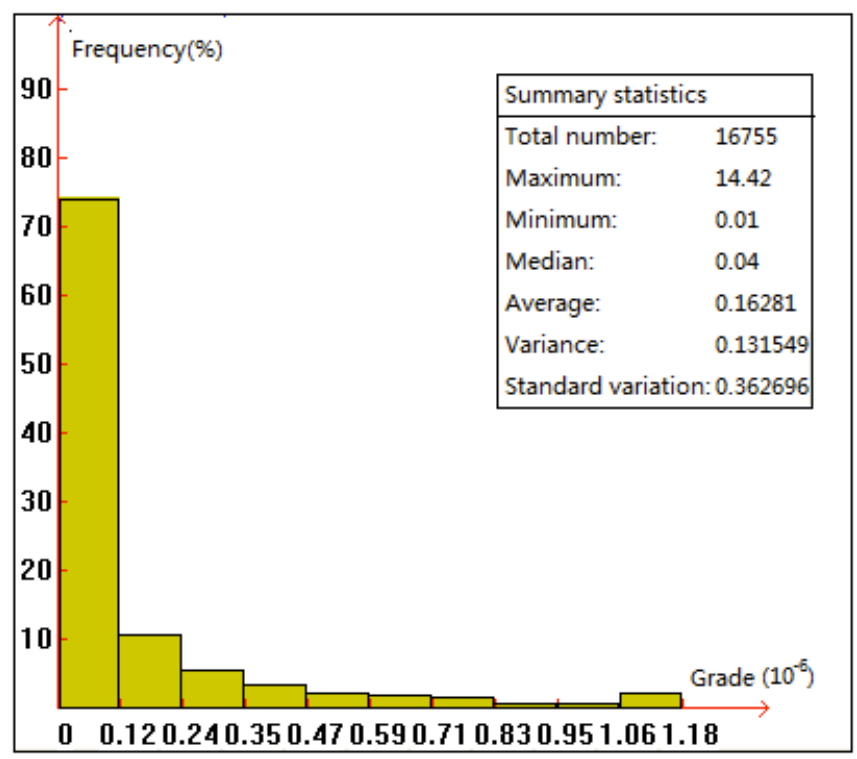

Fig. (3). Basic statistic information of the sample grades.

(2) Threshold determination and indicator processing of the grade data

For the traditional IK method, in order to find out a proper series of threshold values, the cumulative probability distribution of sample grades within the study area needs to be observed and analyzed in detail. In addition, considering the computation speed and efficiency, the cumulative probability distribution function is uniformly divided into ten segments, $\{0.1,0.2,0.3,0.4,0.5,0.6,0.7,0.8,0.9\}$, and thus results in a set of cutoff values, which are then respectively used to transform original sample grades into indicator values.
For the optimized IK method, only the median of the sample grades as a referenced cutoff value is employed to calculate the indicator spatial variation.

\section{(3) Calculation and fitting for spatial variation}

For the all of the indicator grades, the experiment variogram and the corresponding theoretical model are respectively calculated and fitted in this step. A typical calculation result is depicted is Fig. (4), which clearly describes the sharp of experimental and theoretical indicator variogram of the median grade.

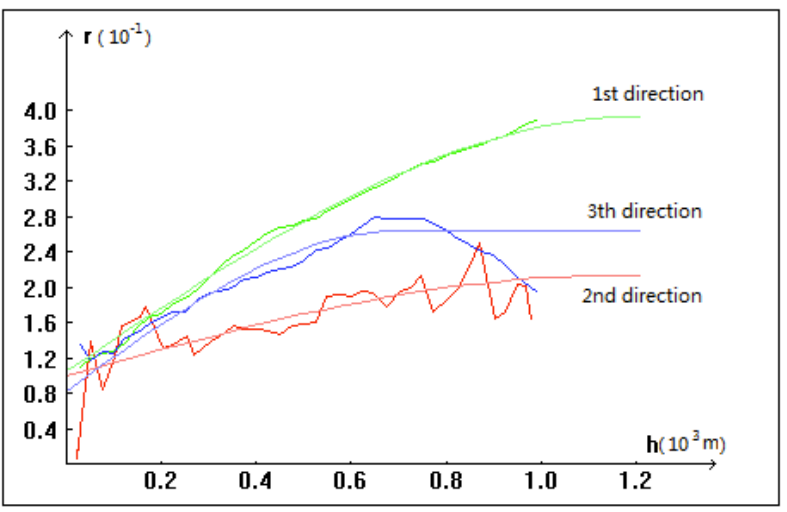

Fig. (4). A typical calculation result of experimental and theoretical indicator variogram.

\section{(4) Ore grade and mineral reserves estimation}

According to basic principles of mineral reserves estimation which have been discussed in the previous sections in this paper, a key step to obtain IK mineral reserves estimates is to interpolate ore grade of block unit in the mineral block model. In this procedure, sample dataset and spatial variation parameters prepared in the above steps are applied in the kriging interpolator (Fig. 5).

Cross validation is carried out to verify the validity of the proposed optimized method. Based on this verification, the optimized IK, traditional IK, OK and IDW method are respectively employed to estimate the grade of mineral block unit. Then a constant weight or density, 2.39, is used to calculate the total metal tonnage of the ore body under study, which produce important data to be compared with actual situations.

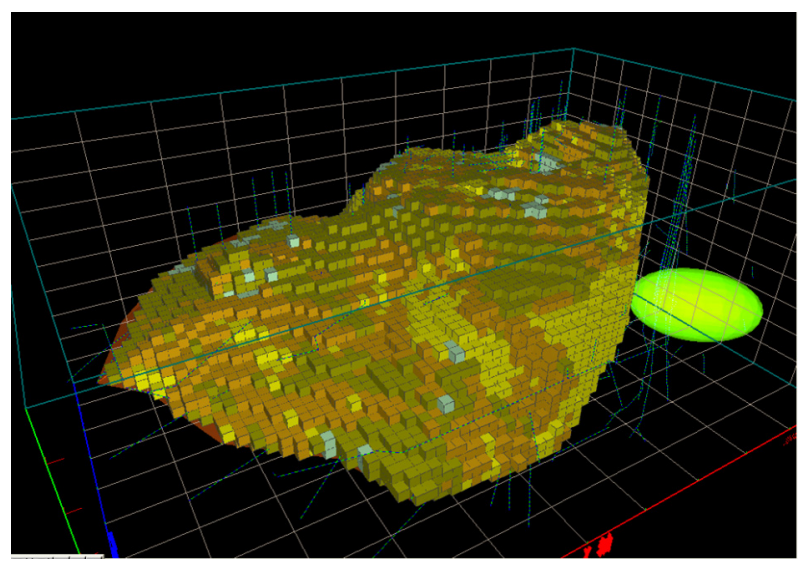

Fig. (5). Typical grade interpolation parameters and result of orebody block model by kriging. 


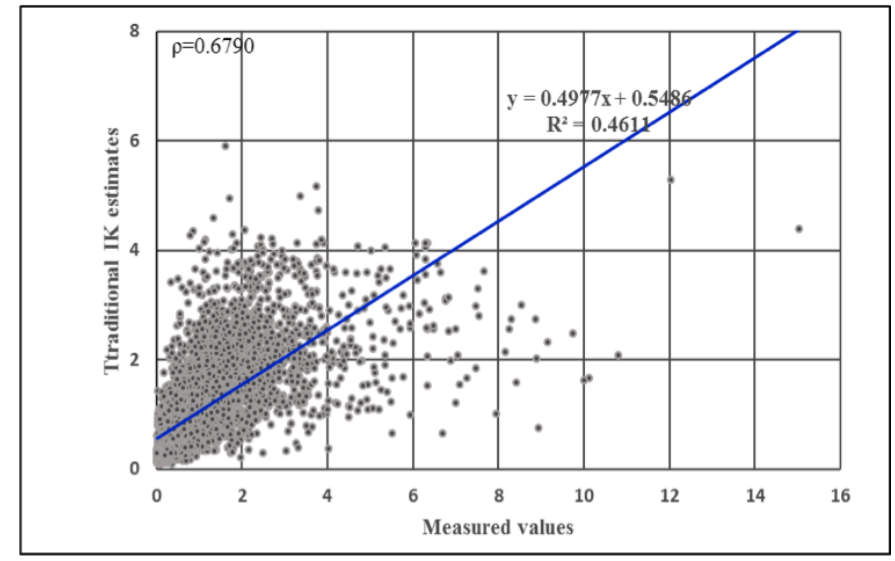

(A)

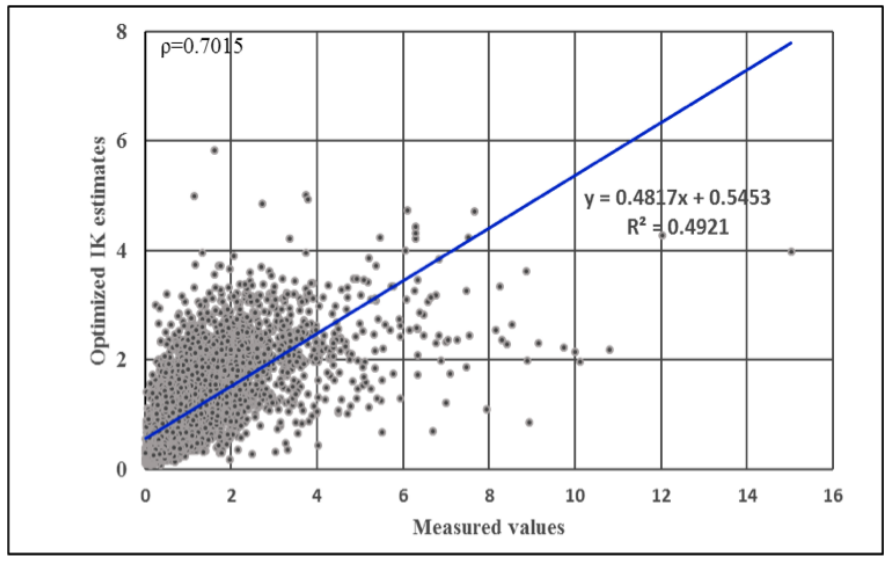

(B)

Fig. (6). Relationship between measured grades and the corresponding estimates resulted from traditional IK (A) and the optimized IK (B) in cross validation.

\subsection{Experimental Results and Analysis}

In the cross validation process, grade estimates resulted from traditional IK and the optimized IK are respectively depicted in Figs. (6A and 6B). From this figure, it is clear that the estimated values resulted from both of the two types of IK methods are reasonably acceptable. Further analyses can result in that the optimized method produces a more reliable set of grade values than the traditional formation in this interpolation procedure since as to the correlation coefficient, 0.70 is obviously bigger than 0.68 . What's more, the estimated RMSE and time consumption of several common interpolation methods listed in Table 1 can also reveal that the optimized IK estimates are more superior to others in accuracy. By the way, the more time consumption during IK interpolation for the optimization method is as expected and acceptable.

Table 1. Root Mean Square Error (RMSE) of estimates and time consumption resulted from cross validation by IDW, OK, traditional IK and the optimized IK, respectively.

\begin{tabular}{|c|c|c|}
\hline Calculation Method & Time Consumption (s) & RMSE \\
\hline \hline IDW & 32 & $0.636 \%$ \\
\hline OK & 216 & $0.716 \%$ \\
\hline Traditional IK & 1033 & $0.522 \%$ \\
\hline Optimized IK & 1109 & $0.510 \%$ \\
\hline
\end{tabular}

Table 2. Metal tonnage and the associated average grade estimates resulted from IDW, OK, traditional IK and the optimized IK, respectively.

\begin{tabular}{|c|c|c|}
\hline Calculation Method & Metal Tonnage (g) & Average Grade $\mathbf{( 1 0}^{-6}$ ) \\
\hline \hline IDW & 7785630 & 0.361813 \\
\hline OK & 6937090 & 0.427518 \\
\hline Traditional IK & 9194430 & 0.360945 \\
\hline Optimized IK & 8045994 & 0.378331 \\
\hline
\end{tabular}

Mineral reserves estimates resulted from varies of interpolation methods are list in Table 2. Comparing with the true situations which the actual metal tonnage and average grade is about $8.3(\mathrm{t})$ and $0.36\left(10^{-6}\right)$, the optimization IK estimate is closest to the real world among the four typical interpolation methods.

Therefore, the optimized IK can be proven valid by comparing with other methods and actual situations.

\section{CONCLUSION}

In theory, IK mineral reserves estimation method can produce reliable estimates even if the original sample grades disobey normal distribution. In practice, however, application of IK is quite complex especially in the determination of cut-off values and the associated spatial variation. Thus it is significant to discuss and research a method to reduce the operation complexity and implement the solution in computer software.

Based on a detailed analysis on basic principles of mineral reserves estimation method and IK itself, this text proposes an alternative method to implement IK reserves estimation in computer software. In this solution, threshold values of IK are dynamically selected according to the participant samples with the searching window. Thus, this method can theoretically improve the operational efficiency as well as reduce complexity of operation comparing with the classical full IK method.

A typical application of the proposed method based on a real developing mine was carried out to examine the practical validation. As a result, the validity of the optimized IK has be strongly proven by comparing with other methods and actual situations.

Thus, the proposed method is worth being applied for mineral reserve estimation process both in theory and practice.

\section{CONFLICT OF INTEREST}

The authors confirm that this article content has no conflict of interest.

\section{ACKNOWLEDGEMENTS}

Declared none. 


\section{REFERENCES}

[1] P. Goovaerts, Geostatistics for Natural Resources Evaluation, New York, 1997, pp. 483.

[2] D. Shepard, "A two-dimensional interpolation function for irregularly-spaced data", In: Proceedings of the $196823^{\text {rd }}$ ACM National Conference, 1986, pp. 517-524

[3] M. Badel, S. Angorani and M.S. Panahi, "The application of median indicator kriging and neural network in modeling mixed population in an iron ore deposit", Computers and Geosciences, vol. 37, pp. 530-540, 2011.

[4] T. Webber, J.F.C.L. Costa and P. Salvadoretti, "Using borehole geophysical data as soft information in indicator kriging for coal quality estimation", International Journal of Coal Geology, vol. 112, pp. 67-75, 2013.

[5] K. Jerosch, M. Schlüter and R. Pesch, "Spatial analysis of marine categorical information using indicator kriging applied to georefer- enced video mosaics of the deep-sea Håkon Mosby Mud Volcano", Ecological Informatics, vol. 1, pp. 391-406, 2006.

[6] A.N. Houston, W. Otten, P.C. Baveye and S. Hapca, "Adaptivewindow indicator kriging: A thresholding method for computed tomography images of porous media", Computers and Geosciences, vol. 54, pp. 239-248, 2013.

[7] B. Lamoureux, N. Mechbal and J. Massé, "A combined sensitivity analysis and kriging surrogate modeling for early validation of health indicators", Reliability Engineering \& System Safety, vol. 130, pp. 12-26, 2014.

[8] E.H. Isaaks, and R.M. Srivastava, An Introduction to Applied Geostatistics, 1989, p. 592 .

[9] A.G. Journel, and C.V. Deutsch, Geostatistical Software Library and Uses Guide, $2^{\text {nd }}$ ed., New York, 1997, p. 335.

[10] B.D. Ripley, Spatial Statistics, New York, 1981, p. 252.

(C) Li and Wang; Licensee Bentham Open.

This is an open access article licensed under the terms of the Creative Commons Attribution Non-Commercial License (http://creativecommons.org/licenses/by-nc/3.0/) which permits unrestricted, non-commercial use, distribution and reproduction in any medium, provided the work is properly cited. 